\title{
Cardiovascular disease in patients with rheumatoid arthritis: impact of classic and disease-specific risk factors
}

\author{
Giulio Cavalli ${ }^{1,2}$, Ennio Giulio Favalli ${ }^{3}$ \\ ${ }^{1}$ Unit of Immunology, Rheumatology, Allergy and Rare Diseases, IRCCS San Raffaele Hospital, Milan, Italy; ${ }^{2}$ Vita-Salute San Raffaele University, \\ Milan, Italy; ${ }^{3}$ Department of Rheumatology, Gaetano Pini Institute, Milan, Italy \\ Correspondence to: Ennio Giulio Favalli, MD. Department of Rheumatology, Gaetano Pini Institute, Via Gaetano Pini, 920122 Milan, Italy. \\ Email: ennio.favalli@gmail.com. \\ Comment on: Crowson CS, Rollefstad S, Ikdahl E, et al. Impact of risk factors associated with cardiovascular outcomes in patients with rheumatoid \\ arthritis. Ann Rheum Dis 2018;77:48-54.
}

Submitted Oct 20, 2018. Accepted for publication Oct 29, 2018.

doi: $10.21037 / \mathrm{atm} .2018 .10 .72$

View this article at: http://dx.doi.org/10.21037/atm.2018.10.72

Chronic inflammation is central to the pathogenesis of atherosclerosis and metabolic syndrome, and promotes both formation and rupture of atherosclerotic plaques, which account for ischemic complications of cardiovascular disease (CVD) $(1,2)$. Pro-inflammatory cytokines and chemokines including interleukin-1 (IL-1) as well as adhesion molecules intercellular adhesion molecule (ICAM) and vascular cell adhesion molecule (VCAM), which are highly expressed in atherosclerotic lesions and on layering endothelial cells, promote leukocyte recruitment, impair vasodilation, and induce oxidative stress and pro-coagulant mediators (3-5).

Patients with immune-mediated diseases are at increased risk of developing cardiac inflammatory conditions (6-10). It is thus not unexpected that, despite remarkable advances in treatment, rheumatoid arthritis (RA) patients tend to endure accelerated atherosclerosis and earlier cardiovascular death compared to the general population (11-13). In the field of RA, CVD risk has been the object of several milestone investigations over time. Main epidemiological studies that underpinned the association between RA and CVD include previous work by Lindhardsen and colleagues (11), who examined the risk of acute myocardial infarction in RA patients and found it as high as that of patients with diabetes mellitus; subsequent epidemiological investigations evaluating multiple comorbidities in RA patients with longstanding disease revealed a $51 \%$ prevalence of a Framingham risk score over 20\%, resulting in increased frequency of cardiovascular ischemic events (14).

Successful prevention of CVD requires reliable identification of patients at risk. However, the importance and relative weight of different CVD risk factors on clinical outcomes in patients with RA does not reflect that observed in the general population: in particular, CVD risk predictions for patients with RA cannot rely on traditional parameters, which provide inaccurate estimates (15-17). A peculiar example of difficulties complicating the CVD risk evaluation of patients with RA is the so-called 'lipid paradox' (18): since serum lipid levels decrease with increasing inflammation (19), patients with RA have lower lipoprotein levels compared to the general population, despite increased CVD risk (20).

A massive study carried out by Crowson and colleagues (21), conducted as part of the larger effort 'Cardiovascular Consortium for Rheumatoid Arthritis' (ATACC-RA), indicates that up to $70 \%$ of CVD events in RA patients could be attributable to a combination of classical CVD risk factors and RA-specific clinical features, such as disease activity and serum rheumatoid factor (RF)/ anti-citrullinated protein antibody (ACPA) positivity. This investigation underlines once more the critical importance of closely controlling both disease activity and cardiovascular risk factors in RA patients, and backs this claim up with the strength on large numbers. Nevertheless, it also raises questions about the 'missing risk', which may account for up to $30 \%$ of cardiovascular events in RA (5).

In this study, 5,638 patients with RA without prior CVD were enrolled in 13 different referral rheumatology centers, spread over 10 European or North American countries. 
Data on CVD risk factors and clinical features of RA were collected at time of enrollment in the study, and patients were then followed up for an average of 5.8 years. During this period of time, 148 men and 241 women developed a CVD event (compositely defined as myocardial infarction, angina, need for revascularization, stroke, clinical signs of peripheral vascular disease, and CVD death). This amounted to a 10 -year cumulative incidence of $20.9 \%$ for male and of $11.1 \%$ for female patients, respectively.

The authors then analyzed the impact of RA-specific and non-RA-specific risk factors, relying on population attributable risk (PAR). Simply put, PAR is the proportion of disease that could have been prevented by eliminating a specific risk factor in a study population, calculated by subtracting the conditional probability of disease among individuals without the risk factor to the global probability of disease (22).

They found that, much alike the general population, male RA patients have a higher burden of CVD risk factors than female counterparts, including hypertension, elevated total cholesterol, and smoking habits. Among traditional CVD risk factors, smoking and hypertension had the highest PAR, followed by total cholesterol, regardless of patient gender. These findings were comparable to those of previous, similar studies, such as the INTERHEART study or the work by Schnohr and colleagues $(23,24)$.

Overall, the authors estimated that up to $70 \%$ of all CVD risk could be attributed to traditional risk factors and RA characteristics combined. Specifically, $49 \%$ of CVD events were attributed to CVD risk factors, whereas $30 \%$ were attributed to RA-specific clinical variables. One of the main findings of this study is indeed that the PAR for RA disease activity and for RA severity, as measured by DAS28 and serum RF/ACPA positivity respectively, were comparable in effect size to the PAR for total cholesterol. Given that the risk of CVD in RA is notoriously attributable to systemic inflammation (25), this finding is not totally unexpected. It is nevertheless highly notable, as it is the importance of RA clinical features in determining CVD outcomes among patients, while also substantiating the need for appropriate prevention strategies: specifically, these findings indicate the critical importance of controlling disease activity in effort to dampen CVD risk among patients with RA.

In addition, this study unveils remarkable genderbased differences in the prevalence of classic as well as RAspecific CVD factors. Specifically, data analysis reveals that male and female patients enrolled in this study exhibited some differences in baseline characteristics: male patients were older and had a higher burden of traditional CVD risk factors, including hypertension and smoking history $(\mathrm{P}<0.001)$, which conferred the highest $\mathrm{PAR}$ in this study. In addition, men had higher levels of CRP $(\mathrm{P}<0.001)$. On the other hand, women had slightly higher total cholesterol and ESR levels; of note, they appeared to be more frequent users of synthetic and biological disease-modifying antirheumatic drugs (DMARDs).

Despite these gender-based differences in the patient baseline characteristics and in the prevalence of many risk factors, the PAR for these risk factors did not differ between the sexes. The fact that no statistically significant differences concerning the risk of developing CVD could be attributed to patient gender in this study should come with no surprise. An obvious confounding factor that is likely to have influenced these findings is the strong female preponderance of RA (26): while CVD tends to occur in older men, RA mostly affects younger women, and in this study there was a clear female preponderance of RA (75.9\%). Furthermore, the specific importance of various CVD risk factors in RA differs that in the general population (15).

Clear strengths of this study are represented by the highly relevant study question (27), together with the considerable sample size and diversity, as the massive study cohort included patients from multiple national backgrounds. In addition, most of the data came either from population-based cohorts or from enrollment of consecutive patients, in effort to increase adherence to real life of the study findings. In these regards, limitations that may limit the generalizability of findings include the likely optimal patient counseling and aggressive treatment of CVD risk at the centers involved in this study, which are leading referral centers for RA. Another, more subtle limitation is that RA characteristics, including disease activity, were pinpointed at the time of study inclusion and not monitored thereafter. This is particularly important since PAR, the statistical measure around which this study pivots, is a function of time that depends on the prevalence of the risk factor (22), which in the case of disease activity is likely to change over time. As RA disease activity may fluctuate over time and upon treatment, pinpointing data on clinical activity may lead to under- or over-estimation of the impact of RA clinical features on the development of CVD events.

Prior to the advent of current therapies and, especially, treatment approaches (28), development of RA marked the onset of a severe, destructive disease leading to a painful, progressive course and to permanent disability. With current, aggressive treatment strategies, low disease activity 
or even remission are within reach and has become realistic goals in most patients $(29,30)$. Prevention of cardiovascular death and related comorbidities is now among the new frontiers in the optimal management of RA. As the work by Crawford and colleagues indicates, appropriate management of CVD risk factors is a critical goal of clinical management in patients with RA, as evidenced by the relevant proportion of CVD risk that remains attributable to classic risk factors. However, the sizeable proportion of CVD risk that is imputable to RA clinical features indicates that disease activity and severity play a role that is nearly as relevant. Undoubtedly, knowledge regarding the impact of various CVD risk factors will bring about new, tailored approaches for CVD risk evaluation and prevention in patients with RA (31).

\section{Acknowledgements}

None.

\section{Footnote}

Conflicts of Interest: The authors have no conflicts of interest to declare.

\section{References}

1. Libby P, Ridker PM, Maseri A. Inflammation and atherosclerosis. Circulation 2002;105:1135-43.

2. Ballak DB, Li S, Cavalli G, et al. Interleukin-37 Treatment of Mice with Metabolic Syndrome Improves Insulin Sensitivity and Reduces Pro-inflammatory Cytokine Production in Adipose Tissue. J Biol Chem 2018;293:14224-36.

3. Peiro C, Lorenzo O, Carraro R, et al. IL-1beta Inhibition in Cardiovascular Complications Associated to Diabetes Mellitus. Front Pharmacol 2017;8:363.

4. Cavalli G, Justice JN, Boyle KE, et al. Interleukin 37 reverses the metabolic cost of inflammation, increases oxidative respiration, and improves exercise tolerance. Proc Natl Acad Sci U S A 2017;114:2313-8.

5. Nagy G, Nemeth N, Buzas EI. Mechanisms of vascular comorbidity in autoimmune diseases. Curr Opin Rheumatol 2018;30:197-206.

6. Cavalli G, Foppoli M, Cabrini L, et al. Interleukin-1 Receptor Blockade Rescues Myocarditis-Associated EndStage Heart Failure. Front Immunol 2017;8:131.

7. De Luca G, Cavalli G, Campochiaro C, et al. Myocarditis: An Interleukin-1-Mediated Disease? Front Immunol
2018;9:1335.

8. Ferrero E, Corti A, Haroche J, et al. Plasma Chromogranin $\mathrm{A}$ as a marker of cardiovascular involvement in ErdheimChester disease. Oncoimmunology 2016;5:e1181244.

9. Cavalli G, Pappalardo F, Mangieri A, et al. Treating LifeThreatening Myocarditis by Blocking Interleukin-1. Crit Care Med 2016;44:e751-4.

10. De Luca G, Campochiaro C, Dinarello CA, et al. Treatment of Dilated Cardiomyopathy With Interleukin-1 Inhibition. Ann Intern Med 2018. [Epub ahead of print].

11. Lindhardsen J, Ahlehoff O, Gislason GH, et al. The risk of myocardial infarction in rheumatoid arthritis and diabetes mellitus: a Danish nationwide cohort study. Ann Rheum Dis 2011;70:929-34.

12. Cavalli G, Tomelleri A, Di Napoli D, et al. Prevalence of Takayasu arteritis in young women with acute ischemic heart disease. Int J Cardiol 2018;252:21-3.

13. Ingegnoli F, Fantini F, Favalli EG, et al. Inflammatory and prothrombotic biomarkers in patients with rheumatoid arthritis: effects of tumor necrosis factor-alpha blockade. J Autoimmun 2008;31:175-9.

14. Balsa A, Lojo-Oliveira L, Alperi-Lopez M, et al. Prevalence of Comorbidities in Rheumatoid Arthritis and Evaluation of Their Monitoring in Clinical Practice: The Spanish Cohort of the COMORA Study. Reumatol Clin 2017. [Epub ahead of print].

15. Crowson CS, Matteson EL, Roger VL, et al. Usefulness of risk scores to estimate the risk of cardiovascular disease in patients with rheumatoid arthritis. Am J Cardiol 2012;110:420-4.

16. Arts EE, Popa C, Den Broeder AA, et al. Performance of four current risk algorithms in predicting cardiovascular events in patients with early rheumatoid arthritis. Ann Rheum Dis 2015;74:668-74.

17. Salaffi F, Carotti M, Di Carlo M, et al. The Expanded Risk Score in Rheumatoid Arthritis (ERS-RA): performance of a disease-specific calculator in comparison with the traditional prediction scores in the assessment of the 10 -year risk of cardiovascular disease in patients with rheumatoid arthrit. Swiss Med Wkly 2018;148:w14656.

18. Myasoedova E, Crowson CS, Kremers HM, et al. Lipid paradox in rheumatoid arthritis: the impact of serum lipid measures and systemic inflammation on the risk of cardiovascular disease. Ann Rheum Dis 2011;70:482-7.

19. Choy E, Sattar N. Interpreting lipid levels in the context of high-grade inflammatory states with a focus on rheumatoid arthritis: a challenge to conventional cardiovascular risk actions. Ann Rheum Dis 2009;68:460-9. 
20. Semb AG, Kvien TK, Aastveit AH, et al. Lipids, myocardial infarction and ischaemic stroke in patients with rheumatoid arthritis in the Apolipoprotein-related Mortality RISk (AMORIS) Study. Ann Rheum Dis 2010;69:1996-2001.

21. Crowson CS, Rollefstad S, Ikdahl E, et al. Impact of risk factors associated with cardiovascular outcomes in patients with rheumatoid arthritis. Ann Rheum Dis 2018;77:48-54.

22. Benichou J. A review of adjusted estimators of attributable risk. Stat Methods Med Res 2001;10:195-216.

23. Schnohr P, Jensen JS, Scharling H, et al. Coronary heart disease risk factors ranked by importance for the individual and community. A 21 year follow-up of 12000 men and women from The Copenhagen City Heart Study. Eur Heart J 2002;23:620-6.

24. Yusuf S, Hawken S, Ounpuu S, et al. Effect of potentially modifiable risk factors associated with myocardial infarction in 52 countries (the INTERHEART study): case-control study. Lancet 2004;364:937-52.

25. Cavalli G, Dinarello CA. Treating rheumatological diseases and co-morbidities with interleukin-1 blocking therapies. Rheumatology (Oxford) 2015;54:2134-44.

Cite this article as: Cavalli G, Favalli EG. Cardiovascular disease in patients with rheumatoid arthritis: impact of classic and disease-specific risk factors. Ann Transl Med 2018;6(Suppl 1):S82. doi: $10.21037 / \mathrm{atm} .2018 .10 .72$
26. Favalli EG, Biggioggero M, Crotti C, et al. Sex and Management of Rheumatoid Arthritis. Clin Rev Allergy Immunol 2018. [Epub ahead of print].

27. Tshomba Y, Cavalli G. Priorities of biomedical research. Int J Cardiol 2017;245:256.

28. Todoerti M, Favalli EG, Iannone F, et al. Switch or swap strategy in rheumatoid arthritis patients failing TNF inhibitors? Results of a modified Italian Expert Consensus. Rheumatology (Oxford) 2018;57:vii42-53.

29. Monti S, Klersy C, Gorla R, et al. Factors influencing the choice of first- and second-line biologic therapy for the treatment of rheumatoid arthritis: real-life data from the Italian LORHEN Registry. Clin Rheumatol 2017;36:753-61.

30. Favalli EG, Raimondo MG, Becciolini A, et al. The management of first-line biologic therapy failures in rheumatoid arthritis: Current practice and future perspectives. Autoimmun Rev 2017;16:1185-95.

31. Selmi C, Kon E, De Santis M, et al. How advances in personalized medicine will change rheumatology. Per Med 2018;15:75-8. 